Objectives: To validate the SIMPLE index for systemic lupus erythematosus (SLE) disease activity assessment in Chinese patients.

Methods: Adult patients (age $\geq 18$ years) who fulfilled the 2013 SLICC criteria for SLE and were followed in the Rheumatology clinics of Tuen Mun Hospital, Hong Kong were recruited in a cross-sectional study. Participants were invited to complete the SIMPLE questionnaire before seeing doctors on the same day. The two laboratory results were supplemented by research nurses. Physicians, who were blinded to the SIMPLE results, were asked to complete disease activity assessment by the SELENA-SLEDAI and physicians' global assessment (PGA) after consultation. Correlation was made between the SIMPLE score and the SLEDAI/PGA scores by Spearman's rank correlation test. Receiver operating characteristic (ROC) analysis was performed to find the best cut-off SIMPLE score that predicted a clinical SLEDAI score of 1-6 (mild SLE activity) and $\geq 7$ (moderate/severe activity).

Results: 364 SLE patients were studied (94\% women; age $45.4 \pm 13.4$ years; disease duration $13.2 \pm 8.0$ years). The proportion of patients having a history of neuropsychiatric and renal disease that required immunosuppressive therapies was $9.3 \%$ and $56 \%$, respectively. At the time of questionnaire completion, $69(19 \%)$ patients had SLEDAI $\geq 6$ and $192(53 \%)$ had SLEDAl 1-5. The mean SLEDAI was $3.04 \pm 2.85$ and PGA score was $0.62 \pm 0.55$. A total of 161 $(44 \%)$ had SDI score $\geq 1$. The mean SIMPLE index was $26.0 \pm 12.9$. SIMPLE index correlated significantly with SLEDAI $(\rho=0.76 ; p<0.001)$ and PGA score $(\rho=0.48 ; p<0.001)$. ROC analysis showed that a SIMPLE index of $>27$ points best predicted a clinical SLEDAI score of 1-6 (area under the curve [AUC] $0.78[0.73-0.84$ ]; sensitivity 0.75 ; specificity 0.71 ), and $>36.8$ points best predicted a clinical SLEDAI score of $\geq 7$ (AUC 0.87[0.69-1.00]; sensitivity 0.88 , specificity 0.85 ).

Conclusion: SIMPLE shows a good correlation with SELENA-SLEDAI and PGA. It is a simple tool that enables patients to self-report disease activity and communicate with the health care team more efficiently.

Disclosure of Interests: None declared

DOI: 10.1136/annrheumdis-2021-eular.3386

\section{POS0770 DIFFERENTIAL CLINICAL PRESENTATION BETWEEN SLE RUPHUS PATIENTS AND SLE NON-RHUPUS PATIENTS: A CASE-CONTROL STUDY}

J. Sanchez-Bautista ${ }^{1}$, Y. Santamaria-Alza ${ }^{2}$, C. Quintero ${ }^{2}$, M. Muñoz $^{2}$, A. Ramírez ${ }^{1}$, C. Muñoz ${ }^{2,3}$, A. L. Vanegas-García ${ }^{2,3}$, G. Vásquez ${ }^{2}$, L. A. González ${ }^{2}{ }^{1}$ Universidad de Antioquia, Internal Medicine, Medellín, Colombia: ${ }^{2}$ Universidad de Antioquia, Rheumatology, Medell ín, Colombia; ${ }^{3}$ Hospital San Vicente Fundación, Rheumatology, Medellin, Colombia

Background: Rhupus is a term that describe the coexistence of systemic lupus erythematosus (SLE) and rheumatoid arthritis (RA). Rhupus patients have symmetrical, erosive arthritis, characteristic SLE manifestations and an array of autoantibodies including rheumatoid factor (RF), anti-cyclic citrullinated peptides (anti-CCP), anti-dsDNA, and anti-Sm. ${ }^{1}$ Joint involvement is common in SLE patients $(90 \%)$, but only $1 \%$ to $9,7 \%$ have rhupus. ${ }^{2}$ Due to its low prevalence, there is still a lack of evidence on its presentation and differences with other SLE patients without rhupus.

Objectives: To examine the rhupus prevalence and to compare the clinical and serological characteristics between rhupus, and non-rhupus SLE patients (SLE with arthritis (SLEa), and SLE without arthritis (SLEwa)).

Methods: We performed a case-control study. Rhupus patients (cases) were matched 1:1 with non-rhupus SLE patients (controls). Descriptive statistics were calculated and rhupus, SLEa, and SLEwa were compared.

Results: A total of 72 patients were included, 24 patients in each group; most were female $(87.5 \%)$. The median age at rhupus onset was 36.5 years (IQR $30-42.5)$. RA preceded rhupus in half of the patients, $29 \%$ were initially diagnosed as SLE, and $21 \%$ were initially diagnosed as rhupus. The median duration of RA was 66 months (IQR 30-144).

In the descriptive analysis of rhupus patients, the most affected joints were the proximal interphalangeal and metacarpophalangeal joints (87.5\%), followed by the wrists $(79.1 \%)$. Regarding immunological findings, RF and anti-CCP were positive in $87.5 \%$ and $50 \%$ of the patients, respectively. As to radiographic findings, the median number of erosions in the hands and wrists was 2 (IQR 0-5) and the median number of joints with narrowed joint space was 7 (IQR: 1.5 - 13.5). In terms of treatment, $95.8 \%$ received corticosteroids (median $10 \mathrm{mg} /$ day), $91.6 \%$ antimalarials, $79.1 \%$ methotrexate, $41.6 \%$ leflunomide, and $25.0 \%$ rituximab.

Patients with rhupus were older and had less renal and hematological involvement than patients with SLEa and SLEwa. SLEa patients had higher seropositivity for anti-RNP and anti-Sm, higher neurological involvement, and higher SLEDAI-2K scores than rhupus patients. Comparisons of other clinical and serological variables between SLE rhupus and non-ruphus patients are shown in table 1 and figure 1.
Table 1. Clinical and serological manifestations in rhupus, articular SLE, and non-articular SLE

\begin{tabular}{|c|c|c|c|c|c|}
\hline Variables & $\begin{array}{l}\text { RHUPUS } \\
\text { M (IQR) }\end{array}$ & $\begin{array}{l}\text { SLE with } \\
\text { arthritis } \\
\text { M (IQR) }\end{array}$ & $\begin{array}{l}\text { SLE without } \\
\text { arthritis } \\
\text { M (IQR) }\end{array}$ & $\begin{array}{l}\text { P value RHUPUS } \\
\text { vs. SLE with } \\
\text { arthritis }\end{array}$ & $\begin{array}{l}\mathrm{p} \text { value } \\
\text { RHUPUS } \\
\text { vs. SLE with- } \\
\text { out arthritis }\end{array}$ \\
\hline $\begin{array}{l}\text { Age (years) M } \\
\text { (IQR) }\end{array}$ & $\begin{array}{l}40(31.5 \\
-49)\end{array}$ & $\begin{array}{l}29(21.5 \\
-45)\end{array}$ & $\begin{array}{l}33(19.5 \\
-37.5)\end{array}$ & 0.043 & 0.013 \\
\hline CPR M (IQR) & $2.3(1-8.5)$ & $\begin{array}{l}1.6 \\
(0.3-3.6)\end{array}$ & $0.9(0.2-2.5)$ & 0.337 & 0.032 \\
\hline $\begin{array}{l}\text { Positive anti-RNP } \\
\quad \mathrm{N}(\%)\end{array}$ & 7 (29.1\%) & $14(58.3 \%)$ & $7(29.1 \%)$ & 0.042 & 1.000 \\
\hline $\begin{array}{l}\text { Positive anti-Sm } \\
\quad \mathrm{N}(\%)\end{array}$ & $5(20.8 \%)$ & $15(62.5 \%)$ & $6(25 \%)$ & 0.003 & 0.500 \\
\hline $\begin{array}{l}\text { SLEDAI - 2K } \\
\text { score M (IQR) }\end{array}$ & $4.5(1-7)$ & $9(2-18)$ & $7(3.5-15)$ & 0.045 & 0.082 \\
\hline
\end{tabular}

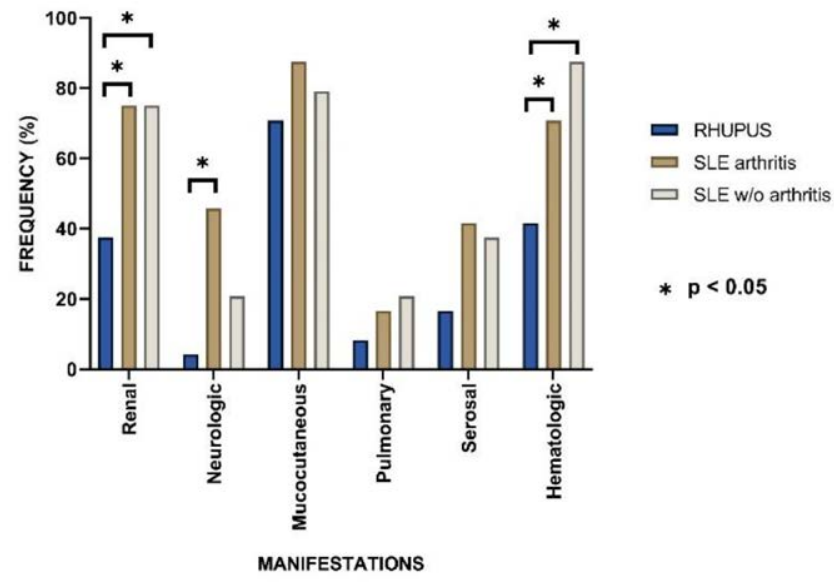

Figure 1. Differences in the frequencies of organic alterations. w/o: without.

Conclusion: Patients with rhupus were older and had lower SLEDAI-2K scores compared with those non-rhupus. Furthermore, renal, neurological, and hematological manifestations were more common in non-rhupus patients than in rhupus patients.

REFERENCES:

[1] Antonini L, et al. Rhupus: a systematic literature review. Autoimmun Rev. 2020;19(9):102612

[2] Tani C, et al. Rhupus syndrome: Assessment of its prevalence and its clinical and instrumental characteristics in a prospective cohort of 103 SLE patients. Autoimmun Rev. 2013; 12:537-41.

Disclosure of Interests: None declared

DOI: 10.1136/annrheumdis-2021-eular.3393

\section{POS0771 VALIDATION OF THE 2019 EUROPEAN LEAGUE AGAINST RHEUMATISM/AMERICAN COLLEGE OF RHEUMATOLOGY (EULAR/ACR) CLASSIFICATION CRITERIA FOR SYSTEMIC LUPUS ERYTHEMATOSUS (SLE) IN HONG KONG CHINESE}

C. C. Mok ${ }^{1}$, Y. K. Chung ${ }^{2}$, C. Lee ${ }^{2}$, L. Y. Ho ${ }^{1}$, C. H. To ${ }^{2} .{ }^{1}$ Tuen Mun Hospital, Medicine, Hong Kong, Hong Kong (SAR); ${ }^{2}$ Pok Oi Hospital, Medicine, Hong Kong, Hong Kong (SAR)

Objectives: To validate the 2019 EULAR/ACR classification criteria for SLE in Hong Kong Chinese patients and compare its performance with the 2012 Systemic Lupus International Collaborating Clinics (SLICC) and 1997 American college of rheumatology (ACR) criteria.

Methods: We retrospectively reviewed the medical records of consecutive patients who attended the Rheumatology clinics in Tuen Mun and Pok Oi hospitals between May and September 2019. Patients with anti-nuclear antibody (ANA) $\geq 1: 80$ were included and patients with ANA $<1: 80$ or no ANA results were excluded. Patients were evaluated and cross-checked for the fulfilment of the 1997 ACR, 2012 SLICC and 2019 EULAR/ACR criteria by two investigators (YKC,CL). Medical records were then reviewed by an expert panel consisting of 3 senior rheumatologists, who were blinded for the results of the criteria evaluation, for a diagnosis of SLE based on the clinical judgement and therapeutic decisions. Teleconferences were arranged by the panel to discuss the discrepancies of the final diagnosis and agreement was made by voting. The three SLE criteria 